\title{
Dose e taxa de aplicação de fungicida no controle da ferrugem da folha (Puccinia triticina) e da mancha amarela (Pyrenophora tritici repentis) do trigo
}

\section{Dose and application rate of fungicide to control leaf rust (Puccinia triticina) and yellow spot (Pyrenophora tritici repentis) of wheat}

\author{
Gustavo Migliorini de Oliveira ${ }^{1 *}$; Diego Dionizio Pereira ${ }^{2}$; \\ Lênio César Moraes de Camargo ${ }^{1}$; Marcelo Gonçalves Balan ${ }^{3}$; \\ Marcelo Giovanetti Canteri ${ }^{4}$; Seiji Igarashi ${ }^{3}$; Otavio Jorge Grigoli Abi Saab ${ }^{4}$
}

\begin{abstract}
Resumo
O objetivo deste trabalho foi estudar a associação entre a dose e a taxa de aplicação, e o efeito desta interação na concentração de fungicida da calda de pulverização, para o controle da ferrugem da folha e mancha amarela do trigo. Realizaram-se dois experimentos, sendo que no primeiro utilizou-se a cultivar CD 104 (suscetível a ferrugem e mancha amarela), tendo como delineamento experimental o fatorial $3 \times 3$ + testemunha sem controle. Os fatores foram dose $\left(0,25,0,30\right.$ e 0,35 L.ha $\left.^{-1}\right)$ e taxa de aplicação $\left(143,286\right.$ e 429 L.ha $^{-1}$ ). Para o segundo experimento usou-se a cultivar BRS 208 (resistente a ferrugem e moderadamente resistente a mancha amarela), tendo como delineamento experimental o fatorial $2 \times 2$ + testemunha sem controle. Os fatores foram dose $\left(0,2\right.$ e 0,3 L.ha $\left.^{-1}\right)$ e taxa de aplicação (143 e 286 L.ha$\left.{ }^{1}\right)$. As aplicações foram realizadas com um pulverizador costal pressurizado via $\mathrm{CO}_{2}$, sob pressão de trabalho de $250 \mathrm{kPa}$, ponta de pulverização XR 110-02, gerando uma taxa de aplicação de $143 \mathrm{~L}^{-h a^{-1}}$. As respectivas taxas de cada tratamento foram alteradas pelo número de vezes que a área foi pulverizada. Utilizou-se ainda o coletor de esporos SIGA associado a dados meteorológicos e de previsão de tempo, que detectou esporos da ferrugem e da mancha amarela antes da efetiva manifestação dos sintomas nas plantas, auxiliando assim na identificação das doenças e na tomada de decisão para a aplicação. Não foi evidenciada nenhuma interação entre dose e taxa de aplicação para nenhum dos experimentos, não havendo, portanto, efeito da concentração de fungicida no controle. A dose e a taxa de aplicação somente exerceram influência no controle da mancha amarela, cujo uso de maiores doses e taxas mostraram-se mais eficientes. Contudo, não foram verificadas diferenças para produtividade e peso hectolitro entre os tratamentos com fungicidas, exceto à testemunha sem aplicação.
\end{abstract}

Palavras-chave: Tecnologia de aplicação, concentração de calda, absorção de fungicida

\begin{abstract}
The aim of this paper was to study the role of dose and rate of application, and the effect of concentration of fungicide in the spray solution resulted from the interaction of these factors, in the control of leaf rust and yellow spot of wheat. It was conducted two experiments, the first used the CD 104 cultivar (susceptible to leaf rust and yellow spot). The experimental design was an factorial $3 \times 3+$ untreated

${ }^{1}$ Eng $^{\text {os }} \mathrm{Agr}^{\text {os }}$, Discente do Curso de Doutorado em Agronomia, Universidade Estadual de Londrina, UEL, Londrina, PR. E-mail: gmigliorini@hotmail.com; lenio_cmc@hotmail.com

2 Eng $^{\circ}$ Agro ${ }^{\circ}$ Graduado, UEL, Londrina, PR. E-mail: diego_dionizio_@hotmail.com

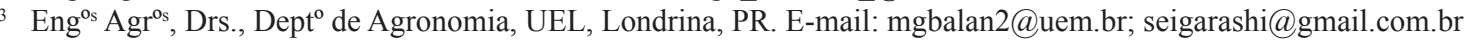

${ }^{4}$ Eng $^{\mathrm{os}} \mathrm{Agr}^{\mathrm{os}}$, Profs. Drs., Dept ${ }^{\mathrm{o}}$ de Agronomia, UEL, Londrina, PR. E-mail: canteri@uel.br; abisaab@uel.br Autor para correspondência
\end{abstract}


control, that involve the factors dose $\left(0,25,0,30\right.$ and $\left.0,35 \mathrm{~L}^{-h^{-1}}\right)$ and application rate $(143,286$ and 429 L.ha ${ }^{-1}$ ). The second experiment used the BRS 208 cultivar (resistant to leaf rust and moderately resistant to yellow spot). The experimental design was an factorial $2 \times 2+$ untreated control, consisting the factors dose $\left(0,2\right.$ and $\left.0,3 \mathrm{~L}_{\mathrm{h}} \mathrm{ha}^{-1}\right)$ and application rate (143 and $\left.286 \mathrm{~L} \cdot \mathrm{ha}^{-1}\right)$. The applications were made with a coastal sprayer by $\mathrm{CO}_{2}$, pressure of $250 \mathrm{kPa}, \mathrm{XR} 110-02$ nozzle, which generated an application rate of $143 \mathrm{~L}_{\mathrm{L}} \mathrm{ha}^{-1}$. The respective rates of each treatment were changed by the number of sprayers per area. It was also used a spore trap denominated Siga, associated with meteorological data and weather forecast, which detected spores of rust and yellow spot before the symptoms in the plants, helping in the identification of disease and in the timing of application. There wasn't any interaction between dose and rate of application for any of the experiments, therefore, there wasn't effect of concentration of fungicide in control. The dose and rate of application just influenced in the control of the yellow spot. Higher doses and rates were more effective. However, no difference was observed for yield and hectolitre weight among treatments, except untreated control.

Key words: Application technology, solution concentration, fungicide absorption

\section{Introdução}

A ocorrência epidêmica da ferrugem da folha (Puccinia triticina Erikss) e mancha amarela do trigo (Pyrenophora tritici-repentis (Died.) Drechsler) tem sido favorecida pelo uso de cultivares sensíveis a estes agentes causais, à presença de inóculo primário no início da safra, e às condições de temperatura e molhamento foliar da cultura, oriundos da presença de orvalho e água de gutação da planta de trigo.

A utilização de fungicidas é um dos principais métodos de controle para tais doenças, apresentando grande efetividade de controle e redução dos níveis de danos e perdas para com a cultura do trigo (DEBONA et al., 2009; KUHNEM JUNIOR et al., 2009; GODOY et al., 2000; PICININI; FERNANDES, 2001).

Entretanto, para que uma aplicação de fungicida atinja sua máxima eficiência de controle, alguns pontos devem ser respeitados, como o momento biológico de controle (COOK; HIMS; VAUGHAN, 1999; FERNANDES; PICININI, 1999) e a tecnologia de aplicação adequada (AZEVEDO, 2007; MENEGHETTI, 2006; PANISSON et al., 2003). No tocante ao momento biológico de controle, o uso de sistemas de previsão, como o coletor de esporos SIGA (GARDIANO et al., 2010; IGARASHI et al., 2008), auxilia na identificação inicial da doença na cultura, no planejamento e tomada de decisão da aplicação de fungicidas e na redução de eventuais contaminações ambientais e outras consequências indesejáveis decorrentes de aplicações desnecessárias (BARRETO; SCALOPPI, 2000).

$\mathrm{Na}$ tecnologia de aplicação, alguns fatores como plantas alvo, fungicida e adjuvante utilizado, taxa de aplicação, tamanho de gotas pulverizadas, ângulo de pulverização, condições meteorológicas durante a pulverização, entre outros, influenciam concomitantemente no nível de controle das doenças. A taxa de aplicação (L.ha ${ }^{-1}$ ) utilizada é o principal fator inerente ao deslocamento das gotas pulverizadas até o alvo, influenciando, assim, nos percentuais de cobertura, penetração e deposição das gotas sobre o dossel da cultura e, por conseguinte, na ação protetora e/ou curativa dos fungicidas (BUTLER ELLIS; WEBB; WESTERN, 2004; MENEGHETTI, 2006; PANISSON et al., 2003; HALLEY et al., 2008; FRITZ et al., 2007).

A dose do produto aplicada é também outro parâmetro a ser considerado. Esta influencia tanto a eficiência de controle - ação fungistática sobre o agente causal objetivado - como também a possibilidade de ocorrência de resistência de fungos aos fungicidas (BRENT, 1995; GHINI; KIMATI, 2000). A combinação entre doses e taxas de aplicação $\left(\right.$ L.ha $\left.^{-1}\right)$ gera concentrações distintas de ingredientes ativos na calda de pulverização, que atuarão na absorção foliar dos fungicidas através da superfície foliar e consequentemente na eficácia 
de controle (AZEVEDO, 2007; WANG; LIU, 2007; KIRKWOOD, 1999).

A absorção é ampliada pelo aumento da cobertura da gota na superfície vegetal, aumento da concentração dos ingredientes ativos e adjuvantes na calda aplicada (FORSTER; ZABKIEWICZ; RIEDERE, 2004, FORSTER; ZABKIEWICZ; LIU, 2006), e pelo uso de massas moleculares menores quando de um mesmo adjuvante (NIELSEN et al., 2005; FORSTER; ZABKIEWICZ; RIEDERE, 2004). Todavia, características inerentes à espessura e o grau de polaridade da camada de cutícula dependentes da espécie vegetal da planta alvo e das condições ambientais em que tais plantas se desenvolvem - influenciam diretamente neste processo de absorção (FERREIRA, 2002, 2003; WANG; LIU, 2007; KIRKWOOD, 1999).

Neste sentido, o objetivo deste trabalho foi estudar a associação entre a dose e a taxa de aplicação e o efeito desta interação na concentração de fungicida da calda de pulverização para o controle da ferrugem da folha e mancha amarela do trigo.

\section{Material e Métodos}

O local de estudo do trabalho foi a fazenda escola da Universidade Estadual de Londrina (2320'30' 'S $51^{\circ} 12^{\prime} 35^{\prime \prime} \mathrm{O}$ ) com altitude aproximada de 560m, na safra de 2010. A classificação climática segundo Köppen é do tipo Cfa (verão quente e chuvoso, precipitação média anual de $1600 \mathrm{~mm}$ ), enquanto o solo da região é classificado como Latossolo Vermelho eutroférrico (EMBRAPA, 1999).

Utilizou-se o coletor de esporos SIGA associado a dados meteorológicos e de previsão de tempo (GARDIANO et al., 2010; IGARASHI et al., 2008) como um sistema de previsão de doenças a título de auxiliar na identificação das doenças antes da manifestação dos sintomas na cultura. Neste coletor foram fixadas lâminas de microscopia óptica com fita adesiva transparente de face dupla para aderência dos esporos presentes no ar. Estas lâminas foram trocadas num intervalo de três dias cada para a identificação dos patógenos realizada por meio de observação em microscópio óptico.

Dois experimentos referentes a diferentes doses e taxas de aplicação do fungicida ciproconazol + azoxistrobina (80 e 200 g. $\mathrm{L}^{-1}$, respectivamente), com adição de $0,5 \%$ v.v. de óleo mineral, foram realizados para dois cultivares distintos quanto à reação às doenças.

Experimento 1 - constituído de um fatorial 3 x 3 + testemunha (sem aplicação de fungicida), envolvendo os fatores dose comercial $(0,25 ; 0,30$ e 0,35 L.ha $\left.^{-1}\right)$ e taxa de aplicação (143; 286 e 429 L.ha' ${ }^{-1}$ ) de fungicida. Para tal utilizou-se o cultivar CD 104, ciclo médio (aproximadamente 120 dias), caracterizado como susceptível à ferrugem da folha (Puccinia triticina), moderadamente susceptível ao oídio (Blumeria graminis) e manchas foliares (Phaeosphaeria nodorum,Cochliobolus sativus, Pyrenophora tritici-repentis), e susceptível à brusone (Magnaporthe grisea) e à giberela (Gibberella zeae). Esta cultivar foi semeada em 23 de abril de 2010, tendo $250 \mathrm{~kg} \cdot \mathrm{ha}^{-1}$ da fórmula 4-148 (NPK) como adubação no momento da semeadura.

Experimento 2 - constituído de um fatorial 2 × 2 + testemunha (sem aplicação de fungicida), sendo o primeiro fator referente à dose comercial $(0,2$ e 0,3 L.ha ${ }^{-1}$ ) e o segundo à taxa de aplicação (143 e 286 L.ha ${ }^{-1}$ ) do fungicida. Para este experimento utilizouse a cultivar BRS 208, caracterizada como resistente à ferrugem da folha ( $P$. triticina), moderadamente resistente ao oídio ( $B$. graminis) e às manchas foliares (P. nodorum, C.sativus, $P$. tritici-repentis), e moderadamente susceptível à brusone (M. grisea) e à giberela (G. zeae). Sua semeadura foi efetuada no dia 19 de abril de 2010, tendo $250 \mathrm{~kg} \cdot \mathrm{ha}^{-1}$ da fórmula 4-14-8 (NPK) como adubação no momento da implantação da cultura.

Em ambos os experimentos as aplicações foram efetuadas por meio de um pulverizador costal pressurizado via $\mathrm{CO}_{2}$, utilizando-se a ponta de pulverização XR 11002, jato plano simples, sob 
pressão de trabalho de $250 \mathrm{kPa}$. As diferentes taxas de aplicações nos experimentos foram obtidas por meio de uma, duas e quatro aplicações consecutivas, a fim de não influenciar no tamanho de gotas pulverizadas em cada tratamento. Reaplicações de fungicida foram realizadas em intervalos de 20 dias cada.

O início das aplicações de fungicida para o experimento 1 ocorreu quando da observação dos primeiros sintomas de ferrugem da folha (estádio de elongação de colmo) e para o experimento 2 dos primeiros sintomas de mancha amarela (estádio de florescimento), baseados na escala fenológica de Zadocks, Ghang e Konzak (1974). Totalizaram-se três aplicações de fungicida para o experimento 1 e duas aplicações para o experimento 2. As características e condições meteorológicas durante as aplicações estão discriminadas na Tabela 1.

Avaliações de severidade da doença foram realizadas periodicamente a cada 7 dias com o auxílio da escala diagramática da EMBRAPA (1999). Foram construídas curvas de progresso das doenças seguido do calculo da AACPD (áreas abaixo da curva de progresso da doença) para a ferrugem da folha ( $P$. triticina) no experimento 1 e para a mancha amarela (P. tritici-repentis) no experimento 2. Foram realizadas ainda no experimento 1 duas avaliações do percentual de severidade de mancha amarela ( $P$. tritici-repentis) nas folhas bandeiras do trigo, decorrente da manifestação tardia da doença.

A estimativa de produtividade e peso hectolitro, padronizados à $13 \%$ de umidade, foram obtidos a partir da colheita manual de $3 \mathrm{~m}^{2}$. Analises de variâncias seguidas do teste Tukey à $5 \%$ de probabilidade foram efetuadas para os tratamentos inerentes aos fatoriais (dose $\mathrm{x}$ taxa de aplicação). Diferenças de cada tratamento isoladamente perante a testemunha sem aplicação foi observada por meio do teste de Dunnet em nível de 5\% de probabilidade.

\section{Resultados e Discussão}

Todas as aplicações de fungicida foram efetuadas no período da manhã, período em que a temperatura, umidade relativa do ar e velocidade do vento $(<4$ $\left.\mathrm{km} \cdot \mathrm{h}^{-1}\right)$ encontravam-se adequadas para a aplicação de fungicida (RAMOS; PIO, 2008). As datas e as condições de temperatura e umidade relativa do ar de cada aplicação encontram-se na Tabela 1.

Tabela 1. Datas e condições meteorológicas observadas durante as aplicações de fungicida dos tratamentos estudados para o controle da ferrugem da folha (P. triticina) e mancha amarela do trigo (P. tritici-repentis), Londrina-PR, 2010.

\begin{tabular}{|c|c|c|c|c|c|c|c|c|c|}
\hline Experimento/ & \multicolumn{3}{|c|}{$1^{\circ}$ Aplicação } & \multicolumn{3}{|c|}{$2^{\circ}$ Aplicação } & \multicolumn{3}{|c|}{$3^{\circ}$ Aplicação } \\
\hline Cultivar & Data & Temp. & UR & Data & Temp. & UR & Data & Temp. & UR \\
\hline $1-\mathrm{CD} 104$ & 09/jun & $24,4^{\circ} \mathrm{C}$ & $77 \%$ & 29/jun & $27,5^{\circ} \mathrm{C}$ & $65 \%$ & 19/jul & $25,4^{\circ} \mathrm{C}$ & $65 \%$ \\
\hline $2-$ BRS 208 & 05/jul & $24,2^{\circ} \mathrm{C}$ & $62 \%$ & 25/jul & $23,5^{\circ} \mathrm{C}$ & $64 \%$ & - & - & - \\
\hline
\end{tabular}

Fonte: Elaboração dos autores.

O coletor de esporos SIGA mostrou-se eficaz como sistema de previsão de doenças ao identificar esporos de doenças de trigo no ar antes de sua infecção efetiva sobre a cultura. Os primeiros esporos de fungos fitopatogênicos observados foram de $P$. triticina em 02 de junho. Transcorridos sete dias observou-se sintomas de ferrugem da folha na cultivar CD 104. Os esporos de $P$. tritici repentis foram observados somente a partir de 22 de junho, sendo que os primeiros sintomas de mancha amarela foram observados treze dias após no cultivar BRS 208 e dezessete dias após no cultivar CD 104. Propágulos de $C$. sativus também foram detectados pelo coletor, porém a mancha marrom não resultou em danos significativos devido a baixa incidência da doença no campo. A Figura 1 apresenta as datas da constatação dos primeiros esporos e sintomas das doenças, associado às condições meteorológicas ocorridas durante a condução dos experimentos. 
Figura 1. Dados diários de precipitação $(\mathrm{mm})$ e temperatura $\left({ }^{\circ} \mathrm{C}\right)$ relacionados ao surgimento dos primeiros esporos e sintomas da ferrugem da folha (P. triticina) e da mancha amarela do trigo (P. tritici-repentis), em Londrina-PR - safra 2010. Fonte: Instituto Agronômico do Paraná - IAPAR.

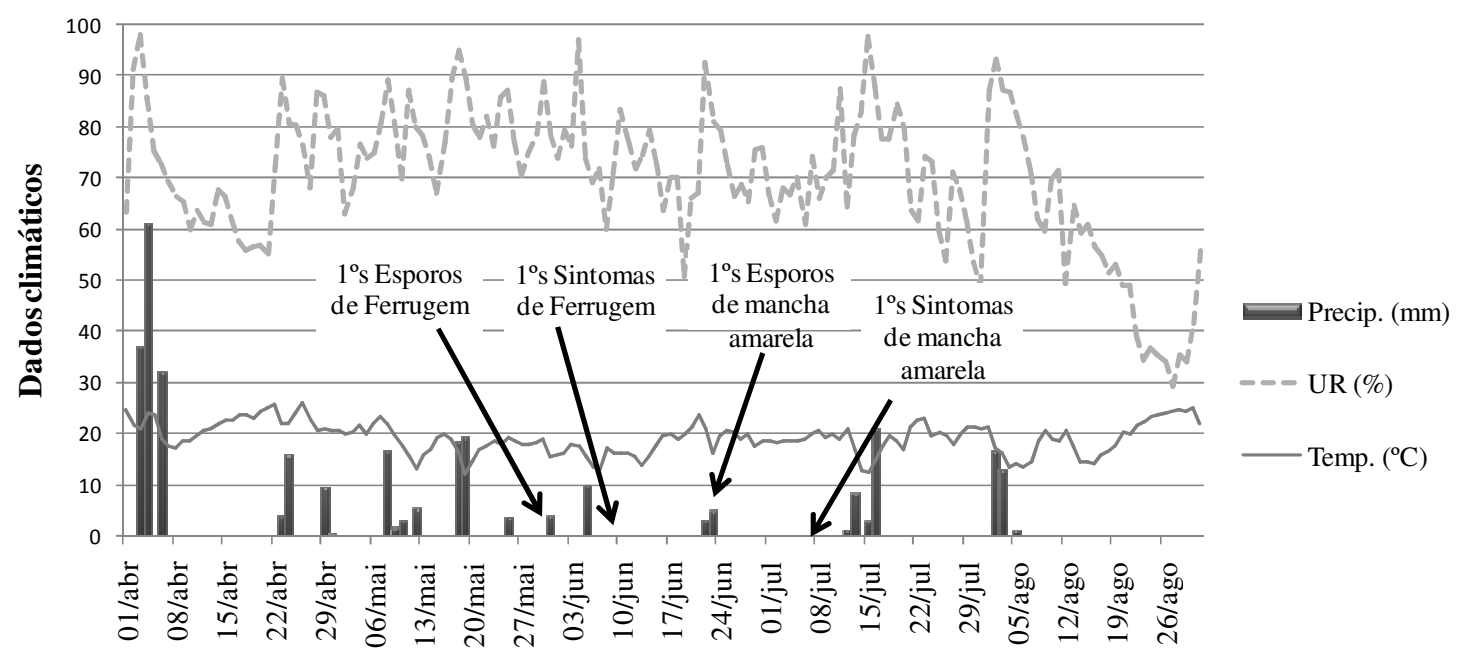

Fonte: Elaboração dos autores.

\section{Experimento 1}

Os fatores dose e taxa de aplicação de fungicida não apresentaram interação significativa $(\mathrm{P}<0,05)$ em nenhuma das variáveis analisadas (Tabela 2).

A análise estatística relativa à área abaixo da curva de progresso da doença (AACPD) da ferrugem da folha não evidenciou diferenças significativas entre as doses ou taxas de aplicação do fungicida, apresentando, somente, efeito da aplicação de fungicida em relação à testemunha (Tabela 3).
A manifestação da mancha amarela ( $P$. triticirepentis) neste experimento 1 foi tardia em virtude das aplicações de fungicida mais antecipadas para o controle da ferrugem da folha. Todavia, a análise estatística acerca da severidade nas folhas bandeira da cultura demonstrou apenas efeito de dose de fungicida no controle da mancha amarela (Tabela 4). Apesar das diferenças serem pequenas entre os tratamentos aplicados, e todos eles diferirem da testemunha sem aplicação, o uso da maior dose de fungicida $\left(0,35\right.$ L.ha $\left.{ }^{-1}\right)$ apresentou redução nos índices de severidade.

Tabela 2. Valores de probabilidade de $\mathrm{F}$ obtidos da analise de variância do fatorial dose $\mathrm{x}$ taxa de aplicação de fungicida para AACPD, severidade da mancha amarela ( $P$. tritici-repentis) na folhas bandeiras nos estádio de grãos leitosos e grãos em massa, produtividade (kg.ha-1) e peso hectolitro (g). Experimento 1 - Cultivar CD 104.

\begin{tabular}{cccccc}
\hline Fonte de variação & AACPD & $\begin{array}{c}\text { Severidade de } \\
\text { mancha amarela }- \\
\text { Grãos leitosos } \\
\operatorname{Pr}>\mathrm{Fc}\end{array}$ & $\begin{array}{c}\text { Severidade de } \\
\text { mancha amarela }- \\
\text { Grãos em massa } \\
\operatorname{Pr}>\mathrm{Fc}\end{array}$ & $\begin{array}{c}\text { Produtividade } \\
\left(\mathrm{kg}^{-1} \mathrm{ha}^{-1}\right)\end{array}$ & $\begin{array}{c}\text { Peso hectolitro } \\
(\mathrm{g})\end{array}$ \\
\cline { 2 - 6 } Dose de fungicida & 0,3786 & 0,2923 & $0,0036^{* *}$ & 0,6004 & $\operatorname{Pr}>\mathrm{Fc}$ \\
Taxa de Aplicação & 0,4412 & 0,8814 & 0,7734 & 0,2997 & $0,39735^{*}$ \\
Dose x Taxa de aplicação & 0,7404 & 0,6251 & 0,9569 & 0,1269 & 0,7342 \\
\hline $\mathrm{CV}(\%)$ & 15,93 & 20,67 & 21,7 & 25,35 & 2,99 \\
\hline
\end{tabular}

Fonte: Elaboração dos autores. 
Tabela 3. Área abaixo da curva de progresso da doença (AACPD) em função da dose $(0,25,0,30$ e 0,35 L.ha-1) e da taxa de aplicação $\left(143,286\right.$ e 429 L.ha $\left.^{-1}\right)$ de fungicida para o controle da ferrugem da folha do trigo (P. triticina). Londrina-PR, 2010.

\begin{tabular}{|c|c|c|c|}
\hline \multirow{2}{*}{ Dose } & 0,25 L.ha $^{-1}$ & 0,30 L.ha-1 & 0,35 L.ha $^{-1}$ \\
\hline & $792,90 a^{*}$ & $733,17 a^{*}$ & $732,03 \mathrm{a}^{*}$ \\
\hline \multirow{2}{*}{ Taxa de aplicação } & 143 L.ha $^{-1}$ & 286 L.ha $^{-1}$ & 429 L.ha $^{-1}$ \\
\hline & $788,40 \mathrm{a}^{*}$ & $742,53 \mathrm{a}^{*}$ & $727,17 a^{*}$ \\
\hline
\end{tabular}

Médias seguidas de mesma letra nas linhas não diferem entre si pelo teste Tukey à $5 \%$ de probabilidade. * Diferem da testemunha sem aplicação pelo teste de Dunnet à 5\% de probabilidade. O coeficiente de variação (CV \%) refere-se à analise de variância do fatorial dose x taxa de aplicação.

Fonte: Elaboração dos autores.

Tabela 4. Severidade da manha amarela trigo (P. tritici-repentis) na folha bandeira em função da dose $(0,25,0,30 \mathrm{e}$ 0,35 L.ha-1) e da taxa de aplicação $\left(143,286\right.$ e 429 L.ha $\left.^{-1}\right)$ de fungicida, nos estádios fenológicos de grãos leitosos e em massa. Londrina-PR, 2010.

\begin{tabular}{|c|c|c|c|}
\hline \multicolumn{4}{|c|}{ Estádio - Grãos leitosos } \\
\hline \multirow{4}{*}{ Taxa de aplicação } & 0,25 L.ha ${ }^{-1}$ & 0,30 L.ha-1 & 0,35 L.ha-1 \\
\hline & $4,50 a^{*}$ & $4,37 \mathrm{a}^{*}$ & $3,53 \mathrm{a}^{*}$ \\
\hline & 143 L.ha $^{-1}$ & 286 L.ha-1 & 429 L.ha $^{-1}$ \\
\hline & $4,21 \mathrm{a}^{*}$ & $4,18 \mathrm{a}^{*}$ & $4,04 \mathrm{a}^{*}$ \\
\hline \multirow[t]{2}{*}{ Testemunha sem controle } & & 20,67 & \\
\hline & \multicolumn{3}{|c|}{ Estádio - Grãos em massa } \\
\hline \multirow{2}{*}{ Dose } & 0,25 L.ha ${ }^{-1}$ & 0,30 L.ha ${ }^{-1}$ & 0,35 L.ha ${ }^{-1}$ \\
\hline & $6,58 a^{*}$ & $7,33 \mathrm{a}^{*}$ & $4,25 b^{*}$ \\
\hline \multirow{2}{*}{ Taxa de aplicação } & 143 L.ha $^{-1}$ & 286 L.ha-1 & 429 L.ha-1 \\
\hline & $6,08 \mathrm{a}^{*}$ & $6,16 a^{*}$ & $5,91 \mathrm{a}^{*}$ \\
\hline Testemunha sem aplicação & & 22 & \\
\hline
\end{tabular}

Médias seguidas de mesma letra nas linhas não diferem entre si pelo teste Tukey à $5 \%$ de probabilidade. ${ }^{*}$ Diferem da testemunha sem aplicação pelo teste de Dunnet à $5 \%$ de probabilidade. O coeficiente de variação (CV \%) refere-se à analise de variância do fatorial dose $\mathrm{x}$ taxa de aplicação.

Fonte: Elaboração dos autores.

As variáveis rendimento, produtividade e peso hectolitro dos grãos não apresentaram diferenças estatísticas $(\mathrm{P}<0,05)$ entre os tratamentos referentes à dose e à taxa de aplicação de fungicida, diferindo estes tratamentos apenas da testemunha sem aplicação (Tabela 5).

\section{Experimento 2}

A análise estatística não verificou interação significativa entre dose e taxa de aplicação de fungicida em nenhuma das variáveis analisadas, conforme pode ser observado na Tabela 6 . 
Tabela 5. Produtividade $\left(\mathrm{kg} \cdot \mathrm{ha}^{-1}\right)$ e peso hectolitro $(\mathrm{g})$ em função da dose $\left(0,25,0,30\right.$ e $\left.0,35 \mathrm{~L}^{-h a^{-1}}\right)$ e da taxa de aplicação $\left(143,286\right.$ e $429 \mathrm{~L}^{-}$ha $\left.^{-1}\right)$ de fungicida para o controle da ferrugem da folha (P. triticina) e mancha amarela do trigo (P. tritici-repentis), Londrina-PR, 2010.

\begin{tabular}{|c|c|c|c|}
\hline \multicolumn{4}{|c|}{ Produtividade $\left(\mathrm{kg} \cdot \mathrm{ha}^{-1}\right)$} \\
\hline \multirow{2}{*}{ Dose } & 0,25 L.ha- ${ }^{-1}$ & 0,30 L.ha- ${ }^{-1}$ & 0,35 L.ha $^{-1}$ \\
\hline & $2003,24 a^{*}$ & $2138,73 \mathrm{a}^{*}$ & $1927,76 a^{*}$ \\
\hline \multirow{2}{*}{ Taxa de aplicação } & 143 L.ha $^{-1}$ & 286 L.ha $^{-1}$ & 429 L.ha-1 \\
\hline & $1967,77 a^{*}$ & $2212,38 a^{*}$ & $1889,57 \mathrm{a}^{*}$ \\
\hline Testemunha sem controle & & 1249,41 & \\
\hline \multicolumn{4}{|c|}{ Peso hectolitro $(\mathrm{g})$} \\
\hline \multirow{2}{*}{ Dose } & 0,25 L.ha $^{-1}$ & 0,30 L.ha-1 & 0,35 L.ha-1 \\
\hline & $69,33 \mathrm{a}$ & $70,41 \mathrm{a}$ & $70,75 \mathrm{a}$ \\
\hline \multirow{2}{*}{ Taxa de aplicação } & 143 L.ha $^{-1}$ & 286 L.ha $^{-1}$ & 429 L.ha-1 \\
\hline & $69,58 \mathrm{a}$ & $69,91 \mathrm{a}$ & $71,10 \mathrm{a}$ \\
\hline Testemunha sem controle & & 69,75 & \\
\hline
\end{tabular}

Médias seguidas de mesma letra nas linhas não diferem entre si pelo teste Tukey à $5 \%$ de probabilidade. ${ }^{*}$ Diferem da testemunha sem aplicação pelo teste de Dunnet à $5 \%$ de probabilidade. O coeficiente de variação (CV \%) refere-se à analise de variância do fatorial dose $\mathrm{x}$ taxa de aplicação.

Fonte: Elaboração dos autores.

Tabela 6. Valores de probabilidade de $\mathrm{F}$ obtidos da analise de variância do fatorial dose $\mathrm{x}$ taxa de aplicação de fungicida para AACPD, produtividade $\left(\mathrm{kg} \cdot \mathrm{ha}^{-1}\right)$ e peso hectolitro (g). Experimento 2 - cultivar BRS 208. LondrinaPR, 2010.

\begin{tabular}{cccc}
\hline Fonte de variação & AACPD & $\begin{array}{c}\text { Produtividade } \\
\left(\mathrm{kg} \cdot \mathrm{ha}^{-1}\right)\end{array}$ & $\begin{array}{c}\text { Peso hectolitro } \\
(\mathrm{g})\end{array}$ \\
& $\mathrm{Pr}>\mathrm{Fc}$ & $\operatorname{Pr}>\mathrm{Fc}$ & 0,2935 \\
Dose de fungicida & $0,0027^{* *}$ & 0,1082 & 0,4267 \\
Taxa de Aplicação & $0,0012^{* *}$ & 0,2731 & 0,6145 \\
\hline Dose x Taxa de aplicação & 0,5623 & 0,7126 & 7,85 \\
\hline CV $(\%)$ & 8,86 & 11,22 & \\
\hline
\end{tabular}

Fonte: Elaboração dos autores.

A variável AACPD, obtida a partir dos níveis de severidade da mancha amarela, evidenciou que o uso da dose 0,3 L.ha- ${ }^{-1}$ e da taxa de aplicação de 286 L.ha $^{-1}$ apresentaram maiores nível de controle de tal doença. Todos os tratamentos que receberam aplicação de fungicida diferiram da testemunha sem controle (Tabela 7).
O rendimento de grãos, a produtividade e o peso hectolitro não foram influenciados pelas doses ou taxa de aplicação dos fungicidas. Diferenças estatísticas foram observadas apenas entre a testemunha sem aplicação e os tratamentos que receberam aplicação de fungicida (Tabela 8). 
Tabela 7. Área abaixo da curva de progresso da doença (AACPD) em função da dose $(0,20$ e 0,30 L.ha-1) e da taxa de aplicação (143 e 286 L.ha $^{-1}$ ) de fungicida no controle da mancha amarela (P. tritici-repentis), Londrina-PR, 2010.

\begin{tabular}{|c|c|c|}
\hline & & \\
\hline \multirow{2}{*}{ Dose } & 0,2 L.ha $^{-1}$ & 0,3 L.ha $^{-1}$ \\
\hline & $559,00 \mathrm{~b}^{*}$ & $454,00 \mathrm{a}^{*}$ \\
\hline \multirow{2}{*}{ Taxa de Aplicação } & 143 L.ha $^{-1}$ & 286 L.ha $^{-1}$ \\
\hline & $550,52 b^{*}$ & $460,50 a^{*}$ \\
\hline Testemunha sem aplicação & & \\
\hline
\end{tabular}

Médias seguidas de mesma letra nas linhas não diferem entre si pelo teste Tukey à $5 \%$ de probabilidade. * Diferem da testemunha sem aplicação pelo teste de Dunnet à $5 \%$ de probabilidade. O coeficiente de variação (CV \%) refere-se à analise de variância do fatorial dose x taxa de aplicação.

Fonte: Elaboração dos autores.

Tabela 8. Produtividade $\left(\mathrm{kg} \cdot \mathrm{ha}^{-1}\right)$ e peso hectolitro $(\mathrm{g})$ em função da dose $\left(0,20\right.$ e $\left.0,30 \mathrm{~L}^{-h a^{-1}}\right)$ e da taxa de aplicação (143 e 286 L.ha $^{-1}$ ) de fungicida para o controle da mancha amarela do trigo (P. tritici-repentis), Londrina-PR, 2010.

\begin{tabular}{|c|c|c|c|c|}
\hline \multirow{3}{*}{ Dose } & \multicolumn{2}{|c|}{ Produtividade $\left(\mathrm{kg} \cdot \mathrm{ha}^{-1}\right)$} & \multicolumn{2}{|c|}{ Peso hectolitro } \\
\hline & 0,2 L.ha ${ }^{-1}$ & 0,3 L.ha ${ }^{-1}$ & 0,2 L.ha-1 & 0,3 L.ha-1 \\
\hline & $3364,06 a^{*}$ & $3591,87 a^{*}$ & $69,55 \mathrm{a}$ & $70,21 \mathrm{a}$ \\
\hline \multirow{2}{*}{ Taxa de Aplicação } & 143 L.ha $^{-1}$ & 286 L.ha-1 & 143 L.ha $^{-1}$ & 286 L.ha' \\
\hline & $3651,99 a^{*}$ & $3303,94 a^{*}$ & $70,35 \mathrm{a}$ & $69,89 \mathrm{a}$ \\
\hline Testemunha sem aplicação & \multicolumn{2}{|c|}{2116,75} & \multicolumn{2}{|c|}{69,92} \\
\hline
\end{tabular}

Médias seguidas de mesma letra nas linhas não diferem entre si pelo teste Tukey à $5 \%$ de probabilidade. * Diferem da testemunha sem aplicação pelo teste de Dunnet à $5 \%$ de probabilidade. O coeficiente de variação $(\mathrm{CV} \%)$ refere-se à analise de variância do fatorial dose x taxa de aplicação.

Fonte: Elaboração dos autores.

\section{Discussão}

Este trabalho evidenciou efeito de doses e taxas de aplicação de fungicida apenas no controle da mancha amarela do trigo (P. tritici-repentis), não influenciando, contudo, nos parâmetros de rendimento da cultura. $\mathrm{O}$ aumento da dose de fungicida promoveu melhoria nos níveis de controle da mancha amarela do trigo em ambos cultivares (CD 104 - susceptível; BRS 208 - moderadamente resistente), assim como o aumento da taxa de aplicação também exerceu influência no controle, porém apenas para a cultivar BRS 208.

Este resultado denota que o controle da mancha amarela do trigo sofreu maior influência dos fatores concernentes à tecnologia de aplicação, mesmo em cultivares moderadamente resistentes (BRS 208).

$\mathrm{O}$ desenvolvimento epidêmico diferenciado da ferrugem da folha (P. triticina) e da mancha amarela (P. tritici-repentis) do trigo é proveniente da agressividade/virulência de cada agente causal, do momento de surgimento dos primeiros esporos e sintomas, da reação distinta de cada cultivar estudado, e das condições meteorológicas ocorridas durante a condução dos experimentos neste ambiente de estudo.

Embora o desenvolvimento inicial da ferrugem da folha (ocorrido a partir de 09 de junho) no cultivar CD 104, bem como da mancha amarela (ocorrido a partir de 05 de junho) no cultivar BRS 208, tenha ocorrido em um período de baixa precipitação pluviométrica (Figura 1), níveis elevados de severidade finais da doença sobre a cultura foram registrados $(90 \%$ e $40 \%$, respectivamente). Isto pode explicado pelo aumento no potencial de inóculo do patógeno decorrente do aumento no número de lesões foliares, pelo aumento da umidade em função da presença de orvalho, característico das condições 
climáticas da região, pela ocorrência da gutação foliar própria da cultura o trigo, como também pelas temperaturas amenas registradas no período. O conjunto destes fatores contribuiu para aumentar a severidade da doença.

O desenvolvimento epidêmico da ferrugem da folha, entre os tratamentos que receberam aplicação de fungicida e a testemunha sem aplicação foi discrepante a partir do dia 17 de junho - oito dias após a primeira aplicação de fungicida (Figura 2).

Já a curva de progresso relativa à mancha amarela do trigo para o experimento 2 (Figura 3) apresentou diferenças entre os tratamentos com aplicação de fungicida e a testemunha sem aplicação a partir do dia 14 de julho - nove dias após a primeira aplicação de fungicida.

Figura 2. Curvas de progresso da doença da ferrugem da folha (P.triticina) em função das doses e taxas de aplicação de fungicida testadas. Londrina-PR, 2010.

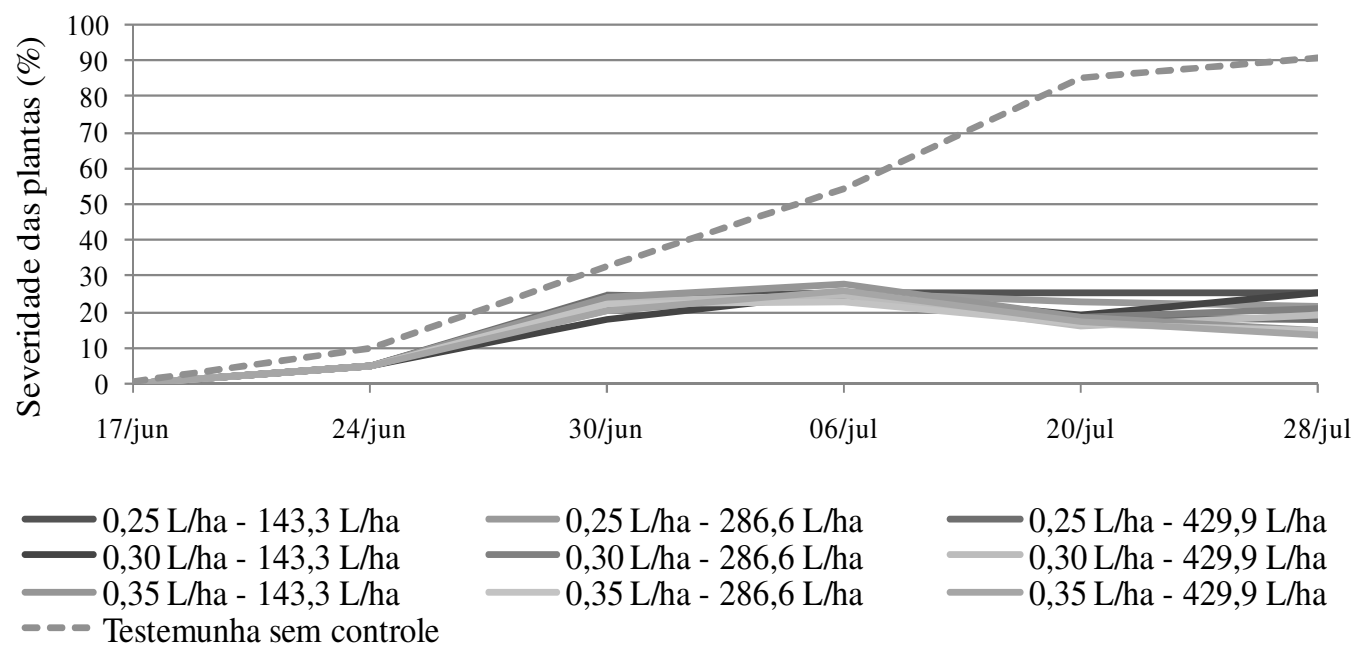

Fonte: Elaboração dos autores.

Figura 3. Curvas de progresso da mancha amarela do trigo (P. tritici-repentis) em função da doses e taxas de aplicação de fungicida testados. Londrina-PR, 2010.

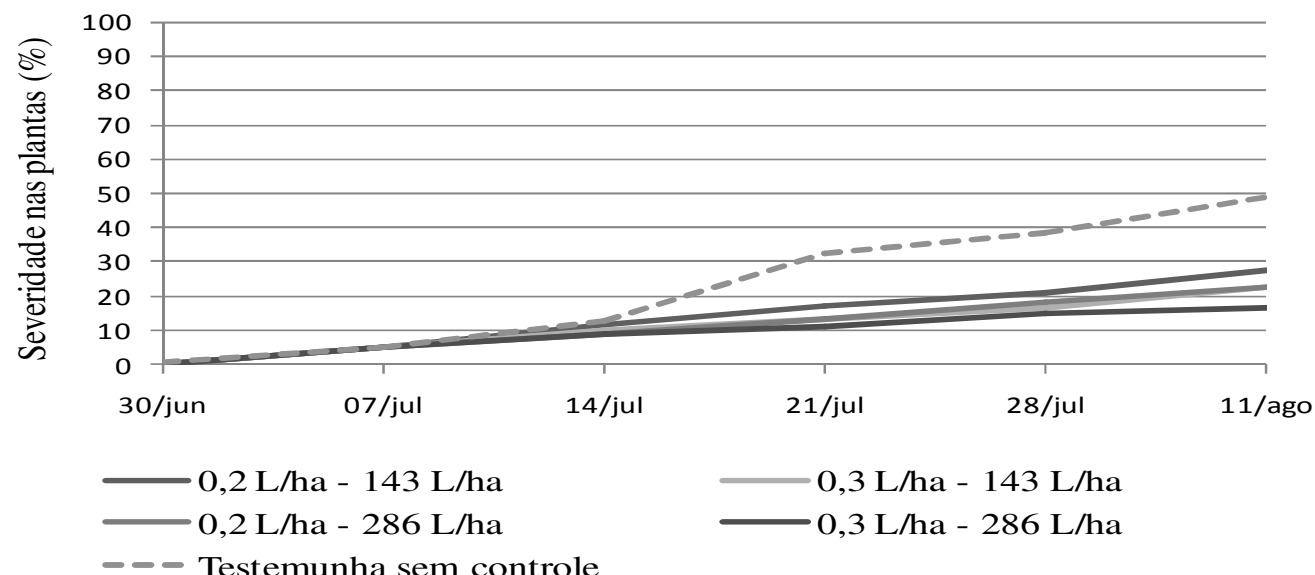

Fonte: Elaboração dos autores. 
As curvas de progresso da doença permitiram concluir que a aplicação de fungicida não inibiu o gradativo aumento da severidade da ferrugem da folha e da mancha amarela de cada experimento, mas contribuiu para reduzir a taxa de desenvolvimento das doenças, provavelmente devido às aplicações terem sido realizadas curativamente, ou seja, após a manifestação dos primeiros sintomas da ferrugem da folha (experimento 1) e da mancha amarela do trigo (experimento 2), onde poderiam haver infecções incubadas (sem manifestação sintomática) nas plantas no momento das primeiras aplicações de fungicida.

Por não ter sido verificada nenhuma interação significativa entre dose e taxa de aplicação de fungicida em ambos os experimentos (Tabelas 2 e 6), concluiu-se que a concentração de fungicida na calda de pulverização não influenciou nenhuma das variáveis analisadas. No entanto, sabe-se que as concentrações de ingrediente ativo de fungicida na calda estão inter-relacionadas à absorção dos fungicidas sistêmicos pela cultura e consequentemente com o nível de controle das doenças. A absorção incrementa-se com: o aumento da cobertura da gota na superfície vegetal, aumento da concentração dos ingredientes ativos (FORSTER; ZABKIEWICZ; RIEDERE, 2004), aumento da concentração dos adjuvantes utilizados (FORSTER; ZABKIEWICZ; LIU, 2006) e o uso de massas moleculares menores quando de um mesmo adjuvante (NIELSEN et al., 2005; FORSTER; ZABKIEWICZ; RIEDERE, 2004).

Todavia, estes trabalhos mencionados anteriormente foram realizados em condições laboratoriais, o que reduziu a influência dos fatores ambientais externos. Tais fatores referem-se principalmente às características ligadas à camada da cutícula da epiderme foliar da planta alvo, que é dependente da espécie vegetal e das condições ambientais (FERREIRA, 2002, 2003; WANG; LIU, 2007; KIRKWOOD, 1999), bem como das características inerentes à tecnologia de aplicação: momento biológico de controle, percentual de cobertura e penetração das gotas pulverizadas no dossel da cultura, propriedades físico-químicas da calda, adjuvantes e ingredientes ativos utilizados, condições metereológicas durante a aplicação, entre outros (FORSTER; ZABKIEWICZ; RIEDERE, 2004; FORSTER; ZABKIEWICZ; LIU, 2006); KNOCHE et al., 1998; KNOCHE; BUKOVAC, 1999, 2000; WANG; LIU, 2007; KIRKWOOD, 1999).

Embora tenha sido evidenciada influência do aumento da dose e da taxa de aplicação, isoladamente, para o controle da mancha amarela do trigo ( $P$. tritici-repentis), pode-se observar pela Figura 4.4 que o aumento das taxas de aplicação associadas ao aumento da dose de fungicida tendem a reduzir os níveis de severidade tanto da ferrugem da folha (CD 104 - experimento 1) como da mancha amarela do trigo (BRS 208 - experimento 2).

Taxas de aplicação maiores resultam necessariamente na redução na concentração de ingrediente ativo na calda de pulverização, porém uma maior quantidade de calda é aplicada por área, o que promove maiores níveis de cobertura pelas gotas sobre a superfície foliar, influenciando também no aumento da absorção de fungicidas (FORSTER; ZABKIEWICZ; RIEDERE, 2004).

Trabalhos de Forcelini et al. (2008) e Oliveira et al. (2007) exemplificam a complexidade no entendimento de todos estes fatores. Estes autores estudaram os efeitos de doses e taxas de aplicação de fungicida no controle da ferrugem asiática da soja (Phakopsora pachyrhizi) e ferrugem da aveia (Puccinia coronata f. sp. avenae), respectivamente. Ambos os trabalhos relataram melhores níveis de controle com o uso de maiores taxas de aplicação, independentemente da dose de fungicida empregada. Eles notaram ainda que maiores doses de fungicida são necessárias quando da utilização de menores taxas de aplicação para o mesmo nível de controle. 
Figura 4. AACPD (área abaixo da curva de progresso da doença) em função da dose e taxa de aplicação de fungicida. A (ferrugem da folha do trigo - CD 104). B (mancha amarela do trigo - BRS 208).

$\mathrm{AACPD}=2302.5556-1.2125 * \mathrm{x}-9271.875 * \mathrm{y}+0.0007 * \mathrm{x} * \mathrm{x}+1.8471 * \mathrm{x} * \mathrm{y}+13641.6667 * \mathrm{y} * \mathrm{y}$
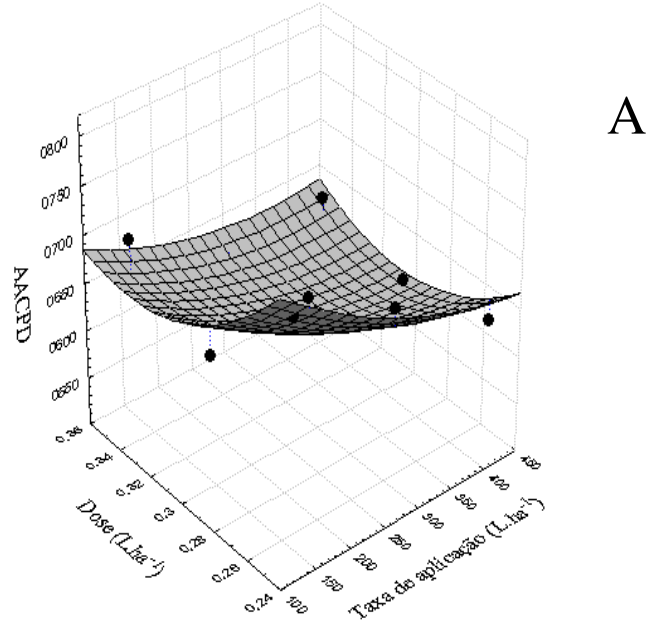

$\mathrm{AACPD}=907-0.642 * \mathrm{x}-1050 * \mathrm{y}$

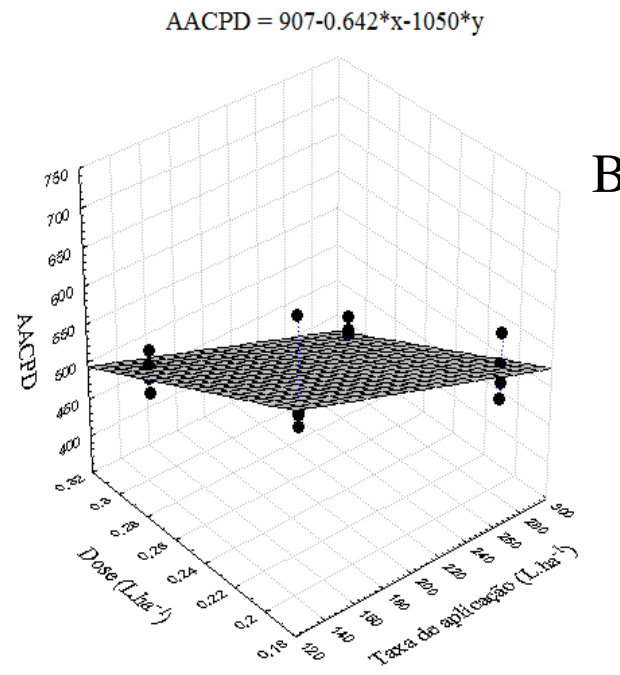

Fonte: Elaboração dos autores.

Neste sentido, infere-se que a concentração de ingrediente ativo na calda de pulverização apresenta menor influência do que a dose e a taxa de aplicação propriamente dita no controle das doenças, pois esta é alterada em função da presença de orvalho diluição - ou pela evaporação natural das gotículas - aumento da concentração (AZEVEDO, 2007; RODRIGUES, 2003).

Acerca das doses de fungicidas, Brent (1995), consultor da FRAC (Fungicide Resistance Action Committee), relata que as doses recomendadas pelo fabricante devem ser empregadas impreterivelmente de forma integral e única. A redução ou aumento da dose, apesar de inicialmente reduzir a velocidade de multiplicação de indivíduos já resistentes, favorece o desenvolvimento da resistência através da mutação e/ou recombinação genética dos indivíduos sobreviventes, tornando-os resistentes a doses superiores no futuro.

Diversos mecanismos estão envolvidos na resistência de fungos a fungicidas, porém estes em sua maioria são pouco conhecidos. O principal deles refere-se às modificações que ocorrem no sitio de ação do fungicida junto ao fungo, diminuindo sua afinidade ao produto. Outros mecanismos de resistência podem estar associados ainda à utilização de rotas metabólicas alternativas em tal sitio de ação; à redução na absorção; ao aumento do fluxo de fungicidas das células dos fungos; a um aumento compensatório na produção da enzima alvo do fungicida; e a detoxicação do fungicida pelos fungos (GHINI; KIMATI, 2000).

Neste sentido, devem-se considerar todos os fatores ambientais envolvidos no desenvolvimento e resistência de uma determinada doença para interpretação dos resultados obtidos em estudos de tecnologia de aplicação, pois existe discrepância entre os resultados relativos aos parâmetros de deposição e/ou cobertura das gotas em relação aos de resistência e eficácia agronômica. Esse argumento é balizado, por exemplo, na melhoria nos níveis de deposição da calda sobre as anteras do trigo quando do uso de duas pontas de pulverização (angulações diferentes) em um mesmo ponto da barra de pulverização (PANISSON et al., 2003), sem resultar, no entanto, em melhoria na eficiência de controle da giberela do trigo (PANISSON; BOLLER; RE4IS, 2004). 
Existe a tendência natural de se reduzir as taxas de aplicação, em decorrência do aumento da eficiência operacional e redução no consumo de água, com o objetivo de diminuir o custo energético para produção agrícola. Porém, o presente estudo demonstra que o aumento na taxa de aplicação pode acarretar melhoria nos níveis de controle de doenças, em especial da mancha amarela, e deve, portanto, ser considerada para um melhor manejo da doença.

\section{Conclusão}

Pôde se concluir que:

- Não existiu efeito da concentração de fungicida na calda de pulverização no controle da ferrugem da folha do trigo (P. triticina) e da mancha amarela do trigo (P. tritici-repentis);

- O aumento das doses de fungicida proporcionou incremento nos níveis de controle da mancha amarela do trigo ( $P$. tritici-repentis) nas cultivares CD 104 e BRS 208, susceptível e moderadamente resistente a tal doença, respectivamente;

- A taxa de aplicação de 286 L.ha-1 reduziu os níveis de severidade da mancha amarela do trigo $(P$. tritici-repentis) somente para a cultivar BRS 208 (moderadamente resistente), não apresentando efeito na cultivar CD 104 (suscetível);

- A aplicação de fungicida, independentemente da dose e da taxa de aplicação, resultou em maiores níveis de controle da ferrugem da folha do trigo (P. triticina) e da mancha amarela do trigo $(P$. tritici-repentis) em relação à testemunha sem aplicação, reduzindo os níveis de danos.

\section{Referências}

AZEVEDO, L. A. S. Fungicidas sistêmicos: teoria e prática. Campinas: EMOPI, 2007. 283 p.

BARRETO, M.; SCALOPPI, E. A. G. Sistemas de previsão de doenças de hortaliças. In: ZAMBOLIM, L. (Ed.) Manejo Integrado de doenças, pragas e plantas daninhas. 2000. p. 196-184.
BRENT, K. J. Resistência a fungicidas em patógenos de plantas cultivadas: como manejá-la? Bruxelas: GCPF, 1995. 54 p. (FRAC Monografia, n. 1).

BUTLER ELLIS, M. C.; WEBB, A.; WESTERN, N. The effect of different spray liquids on the foliar retention of agricultural sprays by wheat plants in a canopy. Pest Management Science, Bognor Regis, v. 60, n. 8, p. 786794, 2004.

COOK, R. J.; HIMS, M. J.; VAUGHAN, T. B. Effects of fungicide spray timing on winter wheat disease control. Plant Pathology, Chichester, v. 48, n. 1, p. 33-50, 1999.

DEBONA, D.; FAVERA, D. D.; CORTE, G. D.; DOMINGUES, L. S.; BALARDIN, R. S. Controle químico da ferrugem da folha em trigo submetidas a diferentes níveis de adubação nitrogenada. Revista da FZVA, Uruguaiana, v. 16, n. 1, p. 52-65, 2009.

EMPRESA BRASILEIRA DE PESQUISA AGROPECUÁRIA - EMBRAPA. Sistema brasileiro de classificação de solos. Brasília: Embrapa-CNPS, 1999. $306 \mathrm{p}$.

FERNANDES, J. M. C.; PICININI, E. C. Controlando as doenças de trigo na hora certa. Passo Fundo: Embrapa Trigo, 1999. 3 p. (Embrapa Trigo. Comunicado Técnico Online, 22). Disponível em: $<$ http://www.cnpt.embrapa. br/biblio/p_co22.htm>. Acesso em: 26 jun. 2010.

FERREIRA, E. A. Estudos anatômicos de folhas de espécies de plantas daninhas de grande ocorrência no Brasil. IV - Amaranthus deflexus, Amaranthus spinosus, Alternanthera tenella e Euphorbia heterophyll. Planta Daninha, Viçosa, MG, v. 21, n. 2, p. 263-271, 2003.

Estudos anatômicos de folhas de plantas daninhas. I - Nicandra physaloides, Solanum viarum, Solanum americanum e Raphanus raphanistrum. Planta Daninha, Viçosa, MG, v. 20, n. 2, p. 159-167, 2002.

FORCELINI, C. A.; BOLLER, W.; CAUS, D. C.; TOREZAN, A. Definição da dose de um fungicida considerado o volume de calda em soja. In: SINTAG SIMPÓSIO INTERNACIONAL DE TECNOLOGIA DE APLICAÇÃO DE AGROTÓXICOS, 4., 2008, Ribeirão Preto. Anais... Ribeirão Preto: [s.n.], 2008.

FORSTER, W. A.; ZABKIEWICZ, J. A.; LIU, Z. Cuticular uptake of xenobiotics into living plants. Part 2: influence of the xenobiotic dose on the uptake of bentazone, epoxiconazole and pyraclostrobin, applied in the presence of various surfactants, into Chenopodium album, Sinapis alba and Triticum aestivum leaves. Pesticide Management Science, Bognor Regis, v. 62, n. 7, p. 664-672, 2006. 
FORSTER, W. A.; ZABKIEWICZ, J. A.; RIEDERE, M. Mechanisms of cuticular uptake of xenobiotics into living plants: 1 . Influence of xenobiotic dose on the uptake of three model compounds applied in the absence and presence of surfactants into Chenopodium album, Hedera helix and Stephanotis floribunda leaves. Pesticide Management Science, Bognor Regis, v. 60, n. 11 p. 1105-1113, 2004.

FRITZ, B. K.; HOFFMAN, W. C.; MARTIN, D. E.; THOMSON, S. J. Aerial application methods for increasing spray deposition on wheat heads. Applied Engineering in Agriculture, St. Joseph, v. 23, n. 6, p. 709715, 2007.

GARDIANO, C. G.; BALAN, M. G.; FALKOSKI FILHO, J.; CAMARGO, L. C. M. de; OLIVEIRA, G. M. de; IGARASHI, W. T.; SUDO, L. T.; IGARSHI, S.; ABI SAAB, O. J. G.; CANTERI, M. G. Manejo químico da ferrugem asiática da soja, baseado em diferentes métodos de monitoramento. Arquivos do Instituto Biológico, São Paulo, v. 77, n. 3, p. 497-504, 2010.

GODOY, C. V.; OLIVEIRA, N. A.; PAIVA, S. B.; BASTOS, H. B. Eficiência do fungicida azoxistrobin no controle da ferrugem da folha e de manchas foliares do trigo. Summa Phytopathologica, Botucatu, v. 26, n. 1, p. 258. 2000. (Resumo).

GHINI, R.; KIMATI, H. Resistência de fungos a fungicidas. Jaguariúna: Embrapa Meio Ambiente, 2000. $78 \mathrm{p}$.

HALLEY, S.; VAN, E. E.; HOFMAN, V.; PANIGRAHI, S.; GU, H. Fungicide deposition measurements by spray volume, drop size, and prayer system in cereal grains. Applied Engineering in Agriculture, St. Joseph, v. 24, n. 1, p. 15-21, 2008.

IGARASHI, W. T.; CAMARGO, L. C. M.; IGARASHI, S.; GARDIANO, C. G.; OLIVEIRA, G. M.; ABI-SAAB, O. J. G. Manejo químico da ferrugem asiática da soja, baseado em diferentes métodos de monitoramento. Fitopatologia Brasileira, Brasília, v. 34, p. 98, 2008. Suplemento.

KIRKWOOD, R. C. Recent developments in our understanding of the plant cuticle as a barrier to the foliar uptake of pesticides. Pesticide Management Science, Bognor Regis, v. 55, n. 1, p. 69-77, 1999.

KNOCHE, M.; BUKOVAC, M. J. Spray application factors and plant growth regulator performance: II Foliar uptake of gibberellic acid and 2,4-D. Pesticide Management Science, Bognor Regis, v. 55, n. 2, p. 166174, 1999.
Spray application factors and plant growth regulator performance: III. Interaction of daminozide uptake, translocation and phytotoxicity in bean seedlings. Pesticide Management Science, Bognor Regis, v. 56, n. 1, p. 43-48, 2000.

KNOCHE, M.; BUKOVAC, M. J.; NAKAGAWA, S.; CRABTREE, G. D. Spray application factors and plant growth regulator performance: I. Bioassays and biological response. Pesticide Management Science, Bognor Regis, v. 54, n. 2, p. 168-178, 1998.

KUHNEM JUNIOR, P. R.; CASA, R. T.; RIZZI, F. P.; MOREIRA, E. N.; BOGO, A. Desempenho de fungicidas no controle de doenças foliares em trigo. Revista de Ciências Agroveterinárias, Lages, v. 8, n. 1, p. 35-42, 2009.

MENEGHETTI, R. C. Tecnologias de aplicação na cultura do trigo. 2006. Dissertação (Mestrado em Agronomia) - Universidade Federal de Santa Maria, Santa Maria, RS.

NIELSEN, C. M.; STEELE, K. D.; FORSTER, J. A.; ZABKIEWICZ. Influence of dose and molecular weight foliar mass uptake of surfactant. New Zealand Plant Protection, v. 58, n. 1, p. 174-178, 2005.

OLIVEIRA, A. R.; BOLLER, W.; FORCELINI, C. A.; BLUM, R.; LOPES, A. Fungicidas, doses e volume de calda no controle da ferrugem da folha da aveia (Puccinia coronata f. sp. avenae). Engenharia Agrícola, Jaboticabal, v. 27, p. 48-55, jan. 2007. Número Especial.

PANISSON, E.; BOLLER, W.; REIS, E. M. Avaliação da deposição de calda em anteras de trigo, para o estudo do controle químico de giberela (Gibberella zeae). Engenharia Agricola, Jaboticabal, v. 24, n. 1, p. 111-120, 2004.

PANISSON, E.; BOLLER, W.; REIS, E. M.; HOFFMANN, L. Modificação de uma barra de pulverização para a aplicação de fungicida em trigo visando o controle de giberela. Ciência Rural, Santa Maria, v. 33, n. 1, p. 13-20, 2003.

PICININI, E. C.; FERNANDES, J. M. C. Controle da ferrugem da folha e da mancha bronzeada da folha em trigo pelo uso de fungicidas no tratamento de sementes. Fitopatologia Brasileira, Brasília, v. 26, n. 1, p. 100-100, 2001.

RAMOS, H. H.; PIO, L. C. Tecnologia de aplicação de produtos fitossanitários. In: ZAMBOLIM, L.; CONCEIÇÃO, M. Z.; SANTIAGO, T. O que engenheiros agrônomos devem saber para orientar o uso de produtos fitossanitários. Viçosa: UFV, 2008. p. 133-200. 
RODRIGUES, J. D. Fisiologia vegetal e sua importância na tecnologia de aplicação de defensivos. Arquivos do Instituto Biológico, São Paulo, v. 65, n. 1-2, p. 59-61, 2003.

WANG, C. J.; LIU, Z. Q. Foliar uptake of pesticides - present status and future challenge. Pesticide Biochemistry and Physiology, v. 87, n. 1, p. 1-8, 2007.
ZADOCKS, J. C.; GHANG, T. T.; KONZAK, C. F. A decimal code for the growth stages of cereals. Weed Research, Oxford, v. 14, p. 415-421, 1974. 